\title{
Flip the coin: IL-7 and IL-7R in health and disease
}

\author{
João T. Barata ${ }^{1^{*}}$, Scott K. Durum², Benedict Seddon ${ }^{3}$
}

${ }^{1}$ Instituto de Medicina Molecular João Lobo Antunes, Faculdade de Medicina, Universidade de Lisboa, Lisbon, Portugal; ${ }^{2}$ Cancer and Inflammation Program, National Cancer Institute, Frederick MD USA; ${ }^{3}$ Institute of Immunity and Transplantation, Division of Infection and Immunity, University College London, UK.

* Correspondence to: João T. Barata, Instituto de Medicina Molecular João Lobo Antunes, Faculdade de Medicina, Universidade de Lisboa, Av. Prof. Egas Moniz, 1649-028 Lisboa, Portugal; Tel: +351217999524; e-mail: joao_barata@medicina.ulisboa.pt 


\begin{abstract}
Interleukin (IL)-7 and its receptor (IL-7R) are critical for $\mathrm{T}$ - and, in the mouse, B-cell development, as well as differentiation and survival of naïve T-cells, and generation and maintenance of memory T-cells. They are also required for innate lymphoid cell development and maintenance, and consequently for the generation of lymphoid structures and barrier defense. Here, we discuss the central role of IL-7 and IL-7R in the lymphoid system and highlight the impacts of its deregulation, placing a particular emphasis on its 'dark side' as a promoter of cancer development. We also explore therapeutic implications and opportunities associated with either positive or negative modulation of the IL-7/IL-7R signaling axis.
\end{abstract}




\section{Introduction}

Interleukin (IL)-7 is a $25 \mathrm{kDa}$ secreted soluble globular protein encoded by the IL7 gene. Its receptor (IL-7R) is a heterodimeric complex consisting of IL-7R $\alpha$ (encoded by IL7R) and the common gamma chain (encoded by IL2RG), shared with the receptors for IL-2,-4,-7,-9,-15 and 21. While it was first characterized as a growth or survival factor for murine T- and B-cell precursors, its central regulatory role throughout the lymphoid system has become increasingly evident. IL-7 is a critical developmental cue at every stage of T-cell development, for both $\alpha \beta$ and $\gamma \delta$ lineages, and for development and survival of naive T-cells as well as generation and maintenance of CD4 and CD8 memory. More recently, the discovery of the innate lymphoid cell family was accompanied by recognition that IL-7 was also essential for development and maintenance of many of its members and consequently for the generation of lymphoid structures and barrier defense mechanisms mediated by these cells. Thus, IL-7 is tightly interwoven in the very fabric of the entire lymphoid system. It is perhaps unsurprising, then, that IL-7 and IL-7R are also implicated in the pathogenesis of different disease states mediated by dysfunctions of lymphoid function. In this review, we examine the physiology and pathophysiology of IL-7 and IL7R, with particular emphasis on their involvement in the promotion of cancer, for which there is increasing evidence, and consider current opportunities and prospects to either exploit or target IL7R-mediated signaling for therapeutic benefit.

\section{IL-7: expression of ligand and receptor}

Insights into the functions of IL-7 are gained by examining where, and by which cells, the cytokine and its receptor are made. Although Il7 mRNA was first detected at high levels in the thymus, more recent studies, and the generation of a number of $I l 7$ reporter systems, reveal that expression occurs in a broad range of tissues. While there is incomplete agreement between different reporter strains about where Il7 can be detected, this likely relates to the relative 
sensitivity of individual reporters coupled with variation in the extent to which IL-7 is actually produced (reviewed in ${ }^{1}$ ). The highest levels of Il7 production appear to be in lymphoid organs such as thymus and lymph nodes (LNs). Perhaps surprisingly they are considerably lower in spleen and bone marrow (BM). However, expression is also readily detectable in numerous non-lymphoid sites, such as intestine, lung, liver ${ }^{2}$ and skin (Fig. 1). In terms of specific cellular sources, early BM chimeras experiments strongly suggested a non-hematopoietic source of IL-7 and this proves to be the case. Cells of both epithelial and endothelial origin were identified as sources. Thymic epithelial cells (TECs) and fibroblastic reticular cells (FRC) in LNs are important sources ${ }^{3,4}$, whereas lymphatic endothelia in $\mathrm{LNs}^{5}$, afferent lymphatics ${ }^{6}$ and bronchus-associated lymphoid tissue of the lung ${ }^{7}$ may account for the high levels of IL-7 found in lymphatic fluid and ultimately blood serum. Il7 is also constitutively expressed by keratinocytes in the skin while expression is inducible in gut epithelia by IFN $\gamma$ signaling. There are also reports of IL-7 expression in the brain, produced by neuronal progenitor cells. Similarly, IL-7R $\alpha$ is broadly expressed throughout the lymphoid system: on B-cell progenitors (but not mature B-cells), innate lymphoid cells, and throughout T-cell development and maturation - though notably absent on selecting thymocytes and effectors cells ${ }^{8,9}$. There are also emerging reports of IL-7R expression in the development of fetally-derived macrophages (http://dx.doi.org/10.1101/534859), while outside of the hematopoietic system, lymphatic endothelial cells express IL-7R $\alpha^{6}$ that is required to drive lymphatic expansion during formation of tertiary lymphoid structures ${ }^{10}$.

\section{Developmental functions and mechanisms of IL-7}

As suggested by IL-7-deficient mice, IL-7 signaling is critical for development and maintenance of the entire lymphoid compartment, including T-lymphocytes, B-lymphocytes, and innate lymphoid cells. IL-7 plays an important role throughout the process of hematopoiesis, facilitating key lineage fate decisions (Fig. 2). While some controversy over the identity of the 
earliest lymphoid progenitor cell remains, the so-called common-lymphoid progenitor is in part characterized by its expression of IL-7R $\alpha^{11}$, and IL-7 signaling is thought to be important for lymphoid lineage specification, directing cells toward B- rather than T-lymphocyte fate ${ }^{12}$. While mature B-cells do not express the IL-7R, development of pre-pro-B cells from common-lymphoid progenitors (CLPs), and subsequent B-cell development in the BM, absolutely requires IL-7 in the mouse (reviewed in ${ }^{13}$ ), whereas in humans IL-7 appears to have a less critical role.

Lymphoid progenitors leave the BM and migrate to the thymus giving rise to numerous lymphoid lineages whose development is dependent on, or influenced by, IL-7 signaling. Thymic NK cells, in contrast to conventional NK cells, depend on IL- $7^{14}$. Pre-T cell progenitors lack expression of CD4 and CD8 surface antigens and are termed double-negative (DN). In the mouse, DN cells are subdivided into four populations (DN1-4) on the basis of expression of CD44 and CD25. Development of $\gamma \delta$ T-cells from DN2 thymocytes is absolutely dependent on IL-7, and strong IL-7 signaling is thought to specifically favor $\gamma \delta$ over $\alpha \beta$ T-cell lineage choice ${ }^{15}$. Developing $\alpha \beta$ T-cells also require IL-7 at DN3 and DN4 stages, whose function can be substituted only in part by $\mathrm{Bcl} 2$ overexpression $^{16}$ and PI3K activation ${ }^{17}$. IL-7 signaling appears to facilitate proliferation and differentiation of these DN stages by regulating cell growth genes such as CD98, and blocking expression of the transcriptional repressor, Bcl-6, in a Stat5-dependent process ${ }^{16}$. In contrast, positive selection of CD4 CD8 double-positive (DP) thymocytes does not require IL-7 signaling, and these cells lack functional IL-7R expression ${ }^{18}$. In mice, IL-7R functional inactivation is achieved by the near complete loss of IL-7R $\alpha$ surface expression coupled with high expression levels of SOCS proteins that inhibit cytokine signal transduction ${ }^{19}$. Cells that successfully pass selection re-express IL-7R in a multistep process dependent upon TCR signaling ${ }^{8}$, and transcription factors Foxo1 ${ }^{20}$, Ets- $1^{21}$ and NF-kB ${ }^{22}$.

More recently, a family of lymphoid cells, innate lymphoid cells (ILCs), has been described that plays important roles in inflammation, immune defense, tissue development and remodeling ${ }^{23}$. 
There are three broad classes of ILCs (groups 1, 2 and 3) whose regulation of effector function parallels that of conventional T-cells, namely Th1, Th2 and Th17-type. IL-7 is critical for development of both ILC2 and ILC3 and IL-7-deficient mice completely lack these subsets. Aside from $\mathrm{T}$ and $\mathrm{B}$ lymphopenia, it was later recognised that IL-7 and IL-7R deficient mice also exhibited profound defects in secondary lymphoid structures, lacking normal sized LNs and Peyer's patches in the gut ${ }^{24}$. It is now recognized that development of these lymphoid structures requires a subset of Rorc-dependent ILC3: lymphoid tissue-inducer cells (LTi). LTi are required to recruit LN organizer cells of stromal origin, together collaborating in the formation of mature lymphoid structures. In IL-7-deficient mice, these structures initially form normally but fail to develop into LNs after birth. It appears that IL-7 signaling in the LTi is required for the final stage of LN formation ${ }^{25,26}$. Indeed, IL-7-driven expansion of LTi is sufficient for ectopic development of lymphoid structures ${ }^{27}$.

\section{Control of immune homeostasis by IL-7}

In contrast to B-cells, T-cells continue to express IL-7R in both naive and memory states, and IL-7 signaling is critical for long-term maintenance of all T-cell populations. IL-7 promotes cell survival by modulating the intrinsic pathway of apoptosis ${ }^{28}$. The idea that competition for homeostatic resources limits the size of the T-cell compartments is as attractive as it is enduring ${ }^{29}$, and there is evidence from mice lacking one or other CD4 or CD8 lineage that IL-7 constitutes one of the key cues controlling the size of the T-cell compartment.

Following activation, T-cells rapidly lose expression of IL-7R $\alpha$. Maintenance of IL-7R expression in peripheral T-cells is dependent on the activity of the FOXO1 transcription factor ${ }^{20}$. The activity of FOXOs is negatively regulated by Akt-mediated phosphorylation that is activated via PI3K by upstream TCR or IL-2 signaling ${ }^{30}$. This effectively uncouples effector cells from IL-7dependent homeostatic control, relying rather on TCR and IL-2 signaling for their survival and 
proliferation. Following the response, while most effector cells will undergo apoptosis, some survive to become long-term memory cells. IL-7 signaling plays a key role in the formation of this memory population. In the absence of antigen, TCR-dependent repression of IL-7R $\alpha$ expression is reversed, and effector T-cells can re-express IL-7R $\alpha$. In some settings at least, cells that re-express IL-7R $\alpha$ are the precursors of the long-term memory cells, suggesting that IL-7 could be instructing effectors to develop into memory cells ${ }^{31}$. However, there is also evidence that effector T-cells are far more predisposed to undergo apoptosis than naïve or memory T-cells. While re-expression of IL-7R can delay their death, the effector pool still undergoes a significant contraction because the effector cells are less able to compete and survive in response to IL-7 than other T-cells ${ }^{9}$. Nevertheless, IL-7 signaling represents a key gateway into the memory T-cell pool, ensuring only the fittest effectors persist. Following establishment of memory, both CD4 $4^{32}$ and CD8 memory Tcells $^{33}$ continue to be dependent on IL-7 for their long-term survival, although CD8 memory cells also have a strong reliance on IL-15. Tissue-resident memory cells were recently recognized as important mediators of immunity in non-lymphoid organs, where they reside and are maintained. These populations do not recirculate and so their dependence on IL-7 for survival ${ }^{34}$ likely explains why epithelial barriers have local sources of IL-7 production. Indeed, dendritic epidermal $\gamma \delta \mathrm{T}$ cells in the skin are also dependent in part on IL-7 for their survival ${ }^{35}$.

There is a growing body of evidence that ILC survival is IL-7-dependent ${ }^{36,37}$ and that this accounts for some features of immune homeostasis that rely on IL-7. ILCs appear to be particularly sensitive to IL-7 levels. Competition for IL-7 with T-cells appears to greatly restrict the size of the ILC compartment ${ }^{37}$, and it is notable that blockade of IL-7 in vivo affects ILCs more rapidly and to a greater extent than other IL-7-dependent populations such as T-cells ${ }^{36}$. Blockade of IL-7 during citrobacter infection compromises the intestinal barrier, and IL-7 induction during infection drives ILC3 expansion required for normal barrier function ${ }^{38}$. Loss of ILC3 in LNs following IL-7 ablation also reveals a hitherto unappreciated role for these cells in regulating lymphocyte 
migration $^{36}$. Thus, ILC function in immune homeostasis appears highly susceptible to perturbations in IL-7 function.

\section{IL-7/IL-7R in the context of chronic inflammation and autoimmunity}

As highlighted above, lack of IL-7/IL-7R-mediated signaling compromises lymphoid development and homeostasis. Its role in supporting normal immunity is evident in mechanisms that promote antiviral immune responses and limit organ toxicity ${ }^{39}$. However, abnormally high or unregulated levels of IL-7 and IL-7R also associate with immunopathology. IL-7 is thought to support aberrant immune activity in autoimmune diseases such as diabetes and multiple sclerosis ${ }^{40}$, and chronic inflammatory diseases such as rheumatoid arthritis, ankylosing spondylitis and inflammatory bowel disease $\mathrm{u}^{41-44}$. Patients with Crohn’s disease and ulcerative colitis display high IL-7 and IL-7R levels, and evidence of colon-specific increased mucosal IL-7R signaling, with colonic IL-7R $\alpha$ expression predicting resistance to anti-TNF therapy ${ }^{42,}{ }^{45}$. Notably, antibodymediated IL-7R blockade reduces human T-cell homing to the gut and colitis in humanized mouse models ${ }^{45}$.

\section{IL-7 and IL-7R in hematological cancers}

\section{IL-7 in T-cell acute lymphoblastic leukemia}

Can IL-7R-mediated signaling promote proliferation to such an extent that it contributes to the development of lymphoid tumors? The in vitro evidence that IL-7, including that produced by $\mathrm{BM}$ and thymic stromal cells, can induce survival and proliferation of T-cell acute lymphoblastic leukemia (T-ALL) cells is extensive (reviewed in ${ }^{46}$ ). In fact, more than $70 \%$ of T-ALL patients 
present with IL-7R-positive blasts ${ }^{47}$ and the response correlates with IL-7R $\alpha$ expression in leukemia cells ${ }^{47,}{ }^{48}$. In contrast to normal thymocytes, IL-7 appears to stimulate T-ALL cells irrespectively of their maturation block, including at the DP stage ${ }^{49}$, although frequency of response may be higher in more immature cases $^{48}$. Correlative data from AKR/J mice, whose thymocytes naturally overexpress IL-7R $\alpha$ and tend to develop thymic T-cell lymphomas ${ }^{50}$, and from T-ALL patients, whose blasts present with evidence of IL-7 consumption ${ }^{51}$, suggest that IL-7mediated signaling contributes to leukemia development in vivo. Importantly, findings from IL-7 transgenic mice ${ }^{52,53}$ and xenotransplant of human T-ALL cells into IL-7-deficient mice ${ }^{51}$ support this possibility.

Several mechanisms increase IL-7R $\alpha$ expression in T-ALL: NOTCH1, a major T-cell oncogene, activates $I L 7 R$ transcriptionally, which in turn is involved in Notch-mediated T-ALL cell maintenance ${ }^{54}$; ZEB2 translocation leads to IL7R transcriptional upregulation ${ }^{55}$; RPL10 R98S mutation upregulates IL-7R $\alpha$ and downstream signaling elements ${ }^{56}$; mutations in DNM2 ${ }^{57}$, which regulates IL-7R $\alpha$ trafficking and surface availability, may impact IL-7R-mediated signaling; and SOCS5 epigenetic silencing upregulates IL-7R $\alpha$ and JAK/STAT signaling ${ }^{58}$.

\section{IL-7 in B-cell acute lymphoblastic leukemia}

IL-7 also induces proliferation in vitro of B-ALL cells ${ }^{59}$ and promotes B-cell tumorigenesis in IL-7 transgenic mice ${ }^{52}$, whereas high levels of $I L 7 R$ have been associated with CNS involvement and relapse in pediatric B-ALL ${ }^{60}$. In addition, IL-7R $\alpha$ is fundamental for B-ALL arising in mice from combined STAT5 activation and PAX5 haploinsufficiency ${ }^{61}$, and B-ALL development in the context of infection exposure and PAX5 deficiency involves a pre-leukemic state that is hypersensitive to IL-7, subsequently originating leukemia by acquiring JAK3 and JAK1 mutations $^{62}$. BLNK/SLP-65 inactivation occurs in human B-ALL, and mouse BLNK-deficient leukemia cells display constitutive JAK3/STAT5 signaling triggered by autocrine IL- $7^{63}$. 
Accordingly, BLNK-deficiency cooperates with constitutive activation of STAT5, a major IL-7 signaling effector, in promoting mouse B-cell leukemogenesis ${ }^{63}$. Ikaros (encoded by IKZF1) is a critical tumor suppressor, often inactivated in B-ALL, whose role in pre-B-cell differentiation relies largely on turning-off IL-7-dependent gene expression ${ }^{64}$, likely by directly repressing $I L 7 R$ and competing with STAT5 DNA-binding ${ }^{65}$. SH2B3 binds JAK3 and regulates pro-B cell homeostasis by decreasing IL-7-triggered JAK/STAT5 signaling. Accordingly, pre-leukemic Bcell progenitors with combined loss of SH2B3 and Tp53 are hyper-responsive to IL-7 on their path to $\mathrm{B}-\mathrm{ALL}^{66}$.

\section{Idiosyncrasies of IL-7-mediated signaling in healthy and leukemic cells}

We will now focus on T-ALL to consider why IL-7 may preferentially benefit leukemia cells at the expense of their normal counterparts (Fig. 3a-d). For one, IL-7 promotes the expansion of TALL cells, but does not overcome their differentiation block ${ }^{67}$. This means that IL-7 positive effects on developmentally-arrested malignant precursors can be 'perpetuated' in the absence of ensuing differentiation checkpoints. By contrast, normal thymocytes benefit from IL-7 transiently but inevitably differentiate and undergo stringent processes of selection that are at the core of Tcell development, against which T-ALL cells are largely protected by their maturation arrest. Additionally, the activation of oncogenes that strongly promote proliferation, such as c-MYC, can often trigger apoptosis - an effect that can be counterbalanced by external cues. MYC is frequently activated in T-ALL ${ }^{68}$, although its ability to drive malignant transformation per se is debated ${ }^{69}$. Pre-leukemic cells with aberrant MYC activation will thus benefit from an IL-7-rich environment that protects them against oncogene-induced apoptosis, offering time for the acquisition of additional mutations. Oncogenic insults suffered by (pre)malignant cells may also shape how they respond to microenvironmental cues. As noted above, different oncogenic insults upregulate IL7R $\alpha$ in T-ALL and pre-leukemic B-cells often are IL-7-hypersensitive. This is especially relevant 
given that (pre)leukemic cells may actively instruct stromal cells to downregulate IL-7 production in specific niches, decreasing IL-7 availability for normal precursors ${ }^{70}$.

IL-7 binding promotes IL-7R $\alpha$ and $\gamma \mathrm{c}$ heterodimerization, leading to JAK1 and JAK3 activation and consequent downstream signaling, most notably STAT1/3/5, PI3K/Akt/mTOR and MEK/ERK pathways. However, T-ALL cells appear to differ from normal T-cells in the recruitment of signaling pathways towards particular cellular responses to IL-7 (Fig. 3e; reviewed in ${ }^{29}$ ). PI3K signaling is involved in IL-7-induced cell cycle progression ${ }^{71}$ without impacting cell survival or Bcl-2 expression ${ }^{72}$ in naïve T-cells. Contrarily, PI3K/Akt/mTOR signaling is essential for IL-7-mediated proliferation and survival of T-ALL cells by promoting both p27 ${ }^{\text {kip } 1}$ downregulation as well as Bcl-2 upregulation ${ }^{73,74}$. In agreement, IL-7-mediated Bcl-2 upregulation in T-ALL cells appears to be STAT5-independent ${ }^{75}$ - again in opposition to the fact that IL-7mediated STAT5 activation in naïve T-cells associates with survival ${ }^{71}$, suggesting that IL-7mediated Bcl-2 upregulation occurs via STAT5 in normal T-cells. Overall, although normal and leukemic T-cells recruit the same signaling axes downstream from IL-7/IL-7R, they may exploit them to achieve different functional outcomes. These differences have potential therapeutic relevance, since PI3K/Akt/mTOR pathway inhibitors should preferentially eliminate IL-7dependent T-ALL cells while being merely cytostatic for peripheral T-cells.

\section{IL7R gain-of-function mutations}

IL7R gene amplification was reported in $\mathrm{T}-\mathrm{ALL}^{76}$, although the consequences for leukemogenesis are unknown. In contrast, $I L 7 R$ mutational activation is a driver of $A L L^{77-82}$. Somatic gain-of-function mutations in IL-7R $\alpha$ lead to constitutive activation of the receptor promoting cell transformation in vitro and tumor formation in vivo. They were identified in around $10 \%$ of $\mathrm{T}$-ALL cases and more rarely in $\mathrm{B}-\mathrm{ALL}^{77,}{ }^{78}$, where they are enriched in particular subgroups, especially in CRLF2-rearranged, Ph-like, IKZF1 N159Y, iAMP21 and PAX5 P80R 
cases $^{78,83}$. Although enriched in patients overexpressing HOXA and $T L X^{77}$ and frequent in early Tcell precursor ALL (ETP-ALL) ${ }^{79}$, IL7R mutations occur in all T-ALL subtypes ${ }^{82}$.

Mutations in $I L 7 R$ are somatic, heterozygous and affect either exon 5 or 6 , falling into four classes (Fig. 4). Type 1a mutations are the most frequent, occur in exon 6, and are insertions or insertions-deletions that include an unpaired cysteine in the extracellular juxtamembranetransmembrane (EJMT) portion of IL-7R $\alpha$, leading to disulfide bond-dependent mutant receptor homodimerization and constitutive, ligand-independent signaling ${ }^{77}$. Type $1 \mathrm{~b}$ mutations also affect exon 6 but insert non-cysteine residues within the transmembrane region closer to the cytosolic domain, including tryptophans, SxxxG-related motifs, or other residues that are suspected or formally shown to lead to ligand-independent IL-7R $\alpha$ dimerization ${ }^{77,84}$. Type 1c mutations impact the EJMT region, similar to type 1a; however, they result in insertion of positively-charged aminoacids that promote $\gamma c$ heterodimerization and IL-7 hyperresponsiveness ${ }^{85}$. Contrary to the others, type 2 mutations are exclusive to B-ALL, affect exon 5 and lead to S185C substitution in the extracellular domain. They confer cytokine-independence, but require TSLPR/CRLF2 and can increase responsiveness to $\mathrm{TSLP}^{78}$.

The ability of mutant IL-7R per se to originate T- or B-ALL in vivo when expressed in hematopoietic progenitors is yet to demonstrate. Nonetheless, retroviral expression of mutant IL7R $\alpha$ in hematopoietic stem and progenitor cells accelerated Notch-induced T-ALL, whereas in CLPs mutant IL-7R $\alpha$ alone caused mature B-cell leukemia/lymphoma - but not B-ALL ${ }^{81}$. In a different study, mutant $I L 7 R$ overexpression in DN thymocytes with inactivation of the Ink4/Arf tumor suppressor, from $\mathrm{Arf}^{--}$mice, induced thymocyte development arrest at an immature stage, eventually leading to ETP-ALL-like disease ${ }^{80}$. Likewise, mutant IL7R cooperated with NRAS G13D in inducing T-ALL from transplanted DN thymocytes ${ }^{86}$. Interestingly, mutant $I L 7 R$ alone caused multisystemic inflammatory disease rather than leukemia ${ }^{86}$. 


\section{IL-7R-mediated signals in resistance to therapy}

IL-7/IL-7R-mediated signaling may be critical for resistance to conventional chemotherapy and to targeted therapeutics. In T-ALL cells, exogenous IL-7 stimulation or mutational activation of IL-7R signaling elements (including the receptor itself) induce glucocorticoid resistance, which can be bypassed using the JAK inhibitor ruxolitinib or MEK, AKT, mTOR, or dual PI3K/mTOR inhibitors $^{76,87}$. Notably, $I L 7 R$ mutation associates with very poor prognosis in relapsed pediatric TALL cases $^{88}$. Additionally, IL-7 promotes resistance to imatinib in Philadelphia-positive B-ALL cells in vitro ${ }^{89}$ and likely contributes to the same phenomenon in vivo ${ }^{90}$. IL-7 may also mediate resistance to the mTOR inhibitor rapamycin ${ }^{91}$, which may be of relevance also for T-ALL ${ }^{73}$.

\section{IL-7 signaling in other hematological tumors}

An oncogenic role of the IL-7-IL-7R signaling axis may extend to other lymphoid tumors. For example, locally-produced IL-7 promotes proliferation, residence and expression of c-Myb and Bcl-2 in cutaneous T-cell lymphoma (CTCL) cells ${ }^{34}$. Other hematological tumors include chronic lymphocytic leukemia, T-cell prolymphocytic leukemia, and Hodgkin's lymphoma ${ }^{59,} 92$.

\section{Potential tumor suppressor functions of IL-7 signaling}

Whereas excessive IL-7/IL-7R signaling is oncogenic, it is possible that, within particular spatial and developmental scenarios, diminished IL-7R-mediated signaling compromises homeostasis to a degree that paradoxically potentiates malignancy. In support of this notion, IL7R $\alpha$ deficiency potentiates lymphomagenesis in p53-null mice by exacerbating telomere erosion in T-cell precursors ${ }^{93}$. In such a scenario, $I L 7 R$ appears to act more in the lines of a tumor suppressor than an oncogene. Likewise, IL-7R signaling prevents premature AID activation during B-cell development ${ }^{94}$, protecting the B-cell genome and potentially preventing leukemogenesis. However, the alternative is that by preventing AID activity and excessive genomic instability, while 
potentiating viability and proliferation, the IL-7/IL-7R axis actually creates the perfect milieu for transformation.

\section{IL-7 and IL-7R in solid tumors}

IL-7 stimulates multiple immune-mediated mechanisms that contribute to the eradication of tumors. We will, however, continue to explore the pathological role of IL-7/IL-7R-mediated signaling, which may extend beyond hematological cancers (Fig. 5). Epithelial cancers can aberrantly express IL-7R $\alpha$ and IL-7 and may thus benefit from their activity ${ }^{95-98}$. Evidently, effects on the immune system, including promoting inflammation, that indirectly contribute to the generation of a pro-tumoral milieu are likely to occur ${ }^{99}$. The involvement of IL-7 and IL-7R in colon chronic inflammation agrees with the observation that patients with metastatic colorectal cancer display high IL-7 levels ${ }^{42}$ and that the cancer tissue itself can secrete IL- $7^{100}$. Notably, high IL-7 and IL-7R expression in tumor tissues of breast and lung cancer patients correlates positively with LN metastasis and poorer survival ${ }^{95,}$ 96, 101, 102. Likewise, high IL-7 expression in prostate cancer $^{103}$ associates with poor prognosis ${ }^{104}$. These data are incompatible with increased T-cell antitumoral activity and rather suggest IL-7/IL-7R pathophysiologically-relevant activation loops in solid tumors. In agreement, $I L 7 R$ gains were reported in lung ${ }^{105}$, pancreatic ${ }^{106}$ and esophageal ${ }^{107}$ carcinomas. Notably, network analysis of recurrently mutated genes revealed IL-7-mediated signaling activation during clonal evolution of breast cancer ${ }^{108}$.

Can IL-7 directly stimulate non-hematopoeitic cancer cells? In vitro studies using cell lines suggest so. IL-7 promoted proliferation ${ }^{101}$, epithelial-mesenchymal transition (EMT) and metastasis $^{97}$ of human breast cancer cells. Likewise, IL-7 stimulated prostate and bladder cancer cell migration, invasiveness and $\mathrm{EMT}^{104,109}$. In lung cancer, IL-7 stimulated the proliferation of tumor cells via cyclin D1 upregulation and appeared to promote tumor growth also in a subcutaneous xenotransplant mouse model ${ }^{110}$. The expression of IL-7R and cyclin D1 in the tumor 
tissue of lung cancer patients correlated positively, and the latter associated with poor prognosis ${ }^{110}$. In a murine orthotopic breast cancer model, IL-7-expressing cancer-associated fibroblasts promoted tumor growth and maintenance of cancer stem-like cells ${ }^{98}$. IL-7 may also be critical in brain tumors, possibly contributing to resistance to cisplatin treatment in glioma ${ }^{111}$. IL-7 contribution to tumor development may further involve promotion of lymphangiogenesis ${ }^{102}$ and osteoclastogenesis $^{112}$.

\section{IL-7 traction for therapeutic purposes}

\section{IL-7 administration in cancer patients}

Animal experiments consistently showed that IL-7 administered pharmacologically could induce T-cell expansion with little toxicity, suggesting IL-7 could have a therapeutic benefit in humans. Over 400 patients have now been treated with IL-7 with few toxicities other than a transient injection site reaction when administered subcutaneously (s.c.). In the earliest trials using non-glycosylated IL-7, anti-IL-7 antibodies were elicited, but this response has apparently been alleviated using glycosylated IL-7 produced by Revimmune (formerly Cytheris Corp).

Cancer patients were the first humans to be treated with IL-7. Although there were no apparent anti-cancer benefits of IL-7, dramatic immunological effects were reported. The first trial included twelve metastatic melanoma and sarcoma patients ${ }^{113}$. Administration s.c. showed a serum half-life of about 12hrs. Injected 8 times, 3 days apart, the highest IL-7 dose elicited a 3-7-fold increase in circulating naïve T-cells, no increase in Tregs and, in some patients, an increase in Bcell progenitors in BM. A subsequent trial in 16 subjects with refractory cancer ${ }^{114}$ confirmed the increase in naïve $\mathrm{T}$ (but not Tregs), showed this was likely due to increased cycling and Bcl-2associated resistance to apoptosis, and demonstrated increased TCR diversity. Decreased Treg frequency was an important distinction from treatments with IL-2, a related $\gamma c$ cytokine, which had the opposite effect. A study in lymphopenic breast cancer patients showed that IL-7 treatment 
before (but not during) chemotherapy increased CD4 counts $^{115}$. A trial in pediatric sarcoma patients combined a tumor vaccine with IL-7, confirming IL-7 induced an increase in T-cells and and decreased Treg frequency, but without anti-cancer benefit ${ }^{116}$. A trial combining IL-7 with the vaccine Provenge in prostate cancer (CITN-03) showed a doubling of PSA-reactive T-cells that could predict a benefit in a sufficiently powered study. IL-7-Fc (NeoImmune Tech/Genexine) was reported to be well-tolerated and to increase T-cells in healthy subjects (AACR 2019 abstract), is in phase 1b/2a trials combined with Temozolomide in glioblastoma, and with the checkpoint inhibitor Atezolizumab (anti-PD-L1) in skin cancers, melanoma, Merkel Cell carcinoma and cutaneous squamous cell cancer, and with Pembrolizumab (anti-PD-1) in triple negative breast cancer. IL-7Fc will be combined with CAR-T in a trial in acute lymphoblastic leukemia. A trial that will combine Revimmune IL-7 with Atezolizumab in urothelial cancer (NCT03513952) will begin accruing soon.

In hematopoietic stem cell transplant patients, recovery of host T-cells after ablation was increased 2-fold by IL-7 in a trial of 12 patients treated for three weeks. Increases were largely in memory T-cells with augmented TCR diversity and in vitro CMV responses ${ }^{114}$. A trial of IL-7 in cord blood transplantation (RevImmune/MD Anderson NCT03600896) is underway.

\section{IL-7 administration in AIDS and other infectious diseases}

AIDS was the first infectious disease to be investigated with IL-7 therapy. In 13 HIVinfected patients who were concurrently treated with anti-retrovirals but had relatively low CD4 Tcell counts, IL-7 increased CD4 and CD8 T-cells ${ }^{117}$. Increases, mainly in naïve and central memory T-cells, were up to 7-fold and counts remained elevated up to 45 weeks after discontinuation of IL7. In 25 HIV-infected patients who were treated with anti-retrovirals, increased central memory Tcells were seen 14 days after a single injection s.c. of IL-7 ${ }^{118,119}$. Concerns that IL-7 could promote HIV replication were not confirmed, since only a transient increase in viral transcripts was 
observed in a fraction of patients. Mucosa-associated invariant T-cells are deficient in HIV patients despite anti-retroviral-treatment. Increases of these cells by 4-5-fold were reported 12 weeks following three s.c. IL-7 injections ${ }^{119}$. IL-7 also elevated T-cell counts in idiopathic (non-HIV) CD4-deficient patients ${ }^{120}$. There are discussions of testing IL-7 in AIDS patients with mycobacterial infection and in patients with multi-drug resistant bacterial infections.

Progressive multifocal leukoencephalopathy (PML) is a severe, rare central nervous system infection caused by JC virus in immunodeficient patients. A single patient with idiopathic CD4 deficiency and PML, after an initial treatment with antivirals, was treated with IL-7 showing a doubling of blood CD4 T-cells and disappearance of viral sequences in cerebrospinal fluid ${ }^{121}$. Subsequent case reports of compassionate use of IL-7 in PML reported CD4 increases and patient improvement $^{122}$.

Sepsis is frequently fatal despite treatment with antibiotics. Recent studies have shown that much of this morbidity results not from the initiating pathogen but from loss of CD4 T-cells and a subsequent rise in secondary infections ${ }^{123}$. IL-7 ex-vivo was shown to greatly increase IFN $\gamma$ production by T-cells from septic patients ${ }^{124}$. A Phase 1 trial of IL-7 in septic patients showed significant improvement in CD4 counts, although subject numbers were too few to determine whether there was a clinical benefit ${ }^{125}$. A larger trial of IL-7 given IV in sepsis patients is planned (NCT03821038). At NCI, IL-7-Fc will be combined with a set of pathogen vaccines in aging patients with cancer to determine if expansion of T-cell repertoire has functional effects.

\section{IL-7R pathway antagonists in the clinic}


As mentioned above, IL-7/IL-7R signaling has been implicated in autoimmune and chronic inflammatory diseases, as well as in cancer. Therapeutic targeting of the pathway could occur at several levels ${ }^{126}$ and a number of clinical trials are underway and planned.

\section{Anti-IL-7R monoclonal antibodies}

Blocking IL-7R with Mabs could blunt effector T-cells but spare Tregs in autoimmune disease. An antagonist anti-IL-7R $\alpha$ Mab (Pfizer), tested in cynomolgus monkeys, blocked IL-7 in T-cells ex-vivo ${ }^{127}$ and is in Phase I trials in type 1 diabetic and multiple sclerosis patients. Another antagonist anti-IL-7R $\alpha$ Mab (GSK), in trial in multiple sclerosis, was halted prematurely for undisclosed reasons. However, a Phase IIa study in primary Sjogren’s syndrome is underway. An antagonist anti- IL-7R $\alpha$ Mab (OSE Immunotherapeutics) was tested in baboons, showed inhibition of a recall DTH response without T-cell depletion ${ }^{128}$, and blocked human T-cell homing to the gut in humanized mice ${ }^{45}$. A Phase I clinical trial is planned in healthy volunteers, aiming for Phase II evaluation in IBD and Sjogren's syndrome. Cytotoxic anti-IL-7R $\alpha$ Mabs, shown to be effective in translational studies in T-cell acute lymphoblastic leukemia ${ }^{129}$, have been developed at NCI (Hixon et al submitted for publication) and will be evaluated in clinical trials by Fannin LLC.

\section{Pharmacological signaling inhibitors}

The IL-7R pathway offers new druggable targets in $\mathrm{ALL}^{126}$ that are beginning to be explored clinically. As mentioned above, ruxolitinib showed therapeutic benefit in translational studies of TALL ${ }^{77,} 130$, especially in ETP-ALL cases ${ }^{48,87}$. St Jude Children’s Research Hospital will incorporate ruxolitinib into front-line therapy for ETP-ALL (NCT03117751). Probably a much broader set of T-ALL (and B-ALL) patients would benefit from treatment with inhibitors of IL-7R signaling. 


\section{Acknowledgements}

We thank M.Morre (Revimmune), R.Hotchkiss (Wash.U.), M.Cheever (U.Wash), R.Gress (NCI), I.Sereti (NIAID), N.Poirier (OSE Immunotherapeutics), C.Foley and A.Veradhachary (Fannin LLC) and E.Schafer (Bayler U.) for sharing information on clinical trials, and M.Fernandes for help with draft figure preparation. JTB is funded by the consolidator grant ERC CoG-648455 from the European Research Council, under the European Union's Horizon 2020 research and innovation programme, and the FAPESP/20015/2014 and PTDC/MECHEM/31588/2017 grants from Fundação para a Ciência e a Tecnologia, Portugal; SKD is funded by the intramural program of the National Cancer Institute, National Institutes of Health, USA and the Children's Cancer Foundation; BS is funded by the MRC (United Kingdom) under Programme Codes U117573801 and MR/P011225/1.

\section{Author Contributions}

JTB, SKD and BS wrote the manuscript and approved its final version.

\section{Conflict of interest disclosures}

SKD is a co-inventor on the patent application "IL-7R-alpha specific antibodies for treating acute lymphoblastic leukemia” filed by the NIH (DHHS). U.S. Patent Application No. 62/238,612. The other authors declare no conflict of interest. 


\section{References}

1. Kim, G.Y., Hong, C. \& Park, J.-H. Seeing is believing: illuminating the source of in vivo interleukin-7. Immune Network 11, 1--10 (2011).

2. Sawa, Y. et al. Hepatic interleukin-7 expression regulates T cell responses. Immunity 30, 447-457 (2009).

3. Moore, N.C., Anderson, G., Smith, C.A., Owen, J.J. \& Jenkinson, E.J. Analysis of cytokine gene expression in subpopulations of freshly isolated thymocytes and thymic stromal cells using semiquantitative polymerase chain reaction. European journal of immunology 23, 922--927 (1993).

4. Link, A. et al. Fibroblastic reticular cells in lymph nodes regulate the homeostasis of naive T cells. Nature immunology 8, 1255-1265 (2007).

5. Onder, L. et al. IL-7-producing stromal cells are critical for lymph node remodeling. Blood 120, 4675-4683 (2012).

6. Iolyeva, M. et al. Interleukin-7 is produced by afferent lymphatic vessels and supports lymphatic drainage. Blood 122, 2271--2281 (2013).

7. Shinoda, K. et al. Thy1+IL-7+ lymphatic endothelial cells in iBALT provide a survival niche for memory T-helper cells in allergic airway inflammation. Proceedings of the National Academy of Sciences of the United States of America 113, E2842--2851 (2016).

8. Sinclair, C., Saini, M., van der Loeff, I.S., Sakaguchi, S. \& Seddon, B. The long-term survival potential of mature $\mathrm{T}$ lymphocytes is programmed during development in the thymus. Science signaling 4, ra77 (2011).

9. Buentke, E. et al. Do CD8 effector cells need IL-7R expression to become resting memory cells? Blood 108, 1949-1956 (2006).

10. Nayar, S. et al. Bimodal Expansion of the Lymphatic Vessels Is Regulated by the Sequential Expression of IL-7 and Lymphotoxin \$lalpha\$1\$beta\$2 in Newly Formed Tertiary Lymphoid Structures. Journal of immunology (Baltimore, Md. : 1950) 197, 1957-1967 (2016).

11. Kondo, M., Weissman, I.L. \& Akashi, K. Identification of clonogenic common lymphoid progenitors in mouse bone marrow. Cell 91, 661--672 (1997).

12. Dias, S., Silva, H., Jr., Cumano, A. \& Vieira, P. Interleukin-7 is necessary to maintain the B cell potential in common lymphoid progenitors. The Journal of experimental medicine 201, 971-979 (2005).

13. Clark, M.R., Mandal, M., Ochiai, K. \& Singh, H. Orchestrating B cell lymphopoiesis through interplay of IL-7 receptor and pre-B cell receptor signalling. Nat Rev Immunol 14, 69-80 (2014).

14. Vosshenrich, C.A.J. et al. A thymic pathway of mouse natural killer cell development characterized by expression of GATA-3 and CD127. Nature immunology 7, 1217--1224 (2006).

15. Shitara, S. et al. IL-7 produced by thymic epithelial cells plays a major role in the development of thymocytes and TCR \Igamma\$\$delta\$+ intraepithelial lymphocytes. Journal of immunology (Baltimore, Md. : 1950) 190, 6173--6179 (2013).

16. Boudil, A. et al. IL-7 coordinates proliferation, differentiation and Tcra recombination during thymocyte beta-selection. Nature immunology 16, 397-405 (2015).

17. Hagenbeek, T.J. et al. The loss of PTEN allows TCR alphabeta lineage thymocytes to bypass IL-7 and Pre-TCR-mediated signaling. The Journal of experimental medicine 200, 883-894 (2004).

18. Seddon, B. \& Zamoyska, R. TCR and IL-7 receptor signals can operate independently or synergize to promote lymphopenia-induced expansion of naive T cells. J Immunol 169, 3752-3759 (2002). 
19. Yu, Q. et al. Cytokine signal transduction is suppressed in preselection double-positive thymocytes and restored by positive selection. Journal of Experimental Medicine 203, 165-175 (2006).

20. Kerdiles, Y.M. et al. Foxo1 links homing and survival of naive T cells by regulating Lselectin, CCR7 and interleukin 7 receptor. Nature immunology 10, 176-184 (2009).

21. Grenningloh, R. et al. Ets-1 maintains IL-7 receptor expression in peripheral T cells. $J$ Immunol 186, 969-976 (2011).

22. Webb, L.V. et al. Survival of Single Positive Thymocytes Depends upon Developmental Control of RIPK1 Kinase Signaling by the IKK Complex Independent of NF-\$kappa\$B. Immunity 50, 348--361.e344 (2019).

23. Serafini, N., Vosshenrich, C.A. \& Di Santo, J.P. Transcriptional regulation of innate lymphoid cell fate. Nat Rev Immunol 15, 415-428 (2015).

24. Adachi, S. et al. Essential role of IL-7 receptor alpha in the formation of Peyer's patch anlage. International immunology 10, 1-6 (1998).

25. Chappaz, S., Grtner, C., Rodewald, H.-R. \& Finke, D. Kit ligand and Il7 differentially regulate Peyer's patch and lymph node development. Journal of immunology (Baltimore, Md. : 1950) 185, 3514--3519 (2010).

26. Coles, M.C. et al. Role of T and NK cells and IL7/IL7r interactions during neonatal maturation of lymph nodes. Proc Natl Acad Sci U S A 103, 13457-13462 (2006).

27. Meier, D. et al. Ectopic lymphoid-organ development occurs through interleukin 7mediated enhanced survival of lymphoid-tissue-inducer cells. Immunity 26, 643--654 (2007).

28. Pearson, C., Silva, A. \& Seddon, B. Exogenous amino acids are essential for interleukin-7 induced CD8 T cell growth. [corrected]. PLoS One 7, e33998 (2012).

29. Mazzucchelli, R. \& Durum, S.K. Interleukin-7 receptor expression: intelligent design. Nat Rev Immunol 7, 144-154 (2007).

30. Xue, H.H. et al. IL-2 negatively regulates IL-7 receptor alpha chain expression in activated T lymphocytes. Proc Natl Acad Sci U S A 99, 13759-13764 (2002).

31. Kaech, S.M. et al. Selective expression of the interleukin 7 receptor identifies effector CD8 T cells that give rise to long-lived memory cells. Nature immunology 4, 1191--1198 (2003).

32. Seddon, B., Tomlinson, P. \& Zamoyska, R. Interleukin 7 and T cell receptor signals regulate homeostasis of CD4 memory cells. Nature immunology 4, 680-686 (2003).

33. Schluns, K.S., Kieper, W.C., Jameson, S.C. \& Lefrancois, L. Interleukin-7 mediates the homeostasis of naive and memory CD8 T cells in vivo. Nature immunology 1, 426-432. (2000).

34. Adachi, T. et al. Hair follicle-derived IL-7 and IL-15 mediate skin-resident memory T cell homeostasis and lymphoma. Nat Med 21, 1272-1279 (2015).

35. Sumaria, N. et al. Cutaneous immunosurveillance by self-renewing dermal gammadelta T cells. The Journal of experimental medicine 208, 505-518 (2011).

36. Yang, J. et al. IL-7-dependent maintenance of ILC3s is required for normal entry of lymphocytes into lymph nodes. The Journal of experimental medicine 215, 1069-1077 (2018).

37. Martin, C.E. et al. Interleukin-7 Availability Is Maintained by a Hematopoietic Cytokine Sink Comprising Innate Lymphoid Cells and T \\& nbsp;Cells. Immunity 47, 171--182.e174 (2017).

38. Zhang, W., Du, J.-Y., Yu, Q. \& Jin, J.-O. Interleukin-7 produced by intestinal epithelial cells in response to Citrobacter rodentium infection plays a major role in innate immunity against this pathogen. Infection and immunity 83, 3213--3223 (2015).

39. Pellegrini, M. et al. IL-7 engages multiple mechanisms to overcome chronic viral infection and limit organ pathology. Cell 144, 601-613 (2011). 
40. Lee, L.F. et al. Anti-IL-7 receptor-alpha reverses established type 1 diabetes in nonobese diabetic mice by modulating effector T-cell function. Proc Natl Acad Sci U S A 109, 1267412679 (2012).

41. Churchman, S.M. \& Ponchel, F. Interleukin-7 in rheumatoid arthritis. Rheumatology (Oxford) 47, 753-759 (2008).

42. Krzystek-Korpacka, M. et al. Elevated systemic interleukin-7 in patients with colorectal cancer and individuals at high risk of cancer: association with lymph node involvement and tumor location in the right colon. Cancer Immunol Immunother 66, 171-179 (2017).

43. Anderson, C.A. et al. Meta-analysis identifies 29 additional ulcerative colitis risk loci, increasing the number of confirmed associations to 47. Nat Genet 43, 246-252 (2011).

44. Gracey, E. et al. IL-7 primes IL-17 in mucosal-associated invariant T (MAIT) cells, which contribute to the Th17-axis in ankylosing spondylitis. Ann Rheum Dis 75, 2124-2132 (2016).

45. Belarif, L. et al. IL-7 receptor influences anti-TNF responsiveness and T cell gut homing in inflammatory bowel disease. J Clin Invest 130, 1910-1925 (2019).

46. Oliveira, M.L., Akkapeddi, P., Ribeiro, D., Melao, A. \& Barata, J.T. IL-7R-mediated signaling in T-cell acute lymphoblastic leukemia: An update. Adv Biol Regul 71, 88-96 (2018).

47. Karawajew, L. et al. Inhibition of in vitro spontaneous apoptosis by IL-7 correlates with bcl-2 up-regulation, cortical/mature immunophenotype, and better early cytoreduction of childhood T-cell acute lymphoblastic leukemia. Blood 96, 297-306. (2000).

48. Maude, S.L. et al. Efficacy of JAK/STAT pathway inhibition in murine xenograft models of early T-cell precursor (ETP) acute lymphoblastic leukemia. Blood 125, 1759-1767 (2015).

49. Barata, J.T. et al. Common gamma chain-signaling cytokines promote proliferation of Tcell acute lymphoblastic leukemia. Haematologica 89, 1459-1467 (2004).

50. Laouar, Y., Crispe, I.N. \& Flavell, R.A. Overexpression of IL-7R alpha provides a competitive advantage during early T-cell development. Blood 103, 1985-1994 (2004).

51. Silva, A. et al. IL-7 contributes to the progression of human T-cell acute lymphoblastic leukemias. Cancer Res 71, 4780-4789 (2011).

52. Rich, B.E., Campos-Torres, J., Tepper, R.I., Moreadith, R.W. \& Leder, P. Cutaneous lymphoproliferation and lymphomas in interleukin 7 transgenic mice. The Journal of experimental medicine 177, 305-316. (1993).

53. Abraham, N. et al. Haploinsufficiency identifies STAT5 as a modifier of IL-7-induced lymphomas. Oncogene 24, 5252-5257 (2005).

54. Gonzalez-Garcia, S. et al. CSL-MAML-dependent Notch1 signaling controls T lineagespecific IL-7R \{alpha\} gene expression in early human thymopoiesis and leukemia. The Journal of experimental medicine 206, 779-791 (2009).

55. Goossens, S. et al. ZEB2 drives immature T-cell lymphoblastic leukaemia development via enhanced tumour-initiating potential and IL-7 receptor signalling. Nat Commun 6, 5794 (2015).

56. Girardi, T. et al. The T-cell leukemia-associated ribosomal RPL10 R98S mutation enhances JAK-STAT signaling. Leukemia 32, 809-819 (2018).

57. Tremblay, C.S. et al. Loss-of-function mutations of Dynamin 2 promote T-ALL by enhancing IL-7 signalling. Leukemia 30, 1993-2001 (2016).

58. Sharma, N.D. et al. Epigenetic silencing of SOCS5 potentiates JAK-STAT signaling and progression of T-cell acute lymphoblastic leukemia. Cancer Sci [Epub ahead of print] (2019).

59. Digel, W. et al. Human interleukin-7 induces proliferation of neoplastic cells from chronic lymphocytic leukemia and acute leukemias. Blood 78, 753-759. (1991). 
60. Alsadeq, A. et al. IL7R is associated with CNS infiltration and relapse in pediatric B-cell precursor acute lymphoblastic leukemia. Blood 132, 1614-1617 (2018).

61. Heltemes-Harris, L.M. et al. Ebf1 or Pax5 haploinsufficiency synergizes with STAT5 activation to initiate acute lymphoblastic leukemia. The Journal of experimental medicine 208, 1135-1149 (2011).

62. Martin-Lorenzo, A. et al. Infection Exposure is a Causal Factor in B-cell Precursor Acute Lymphoblastic Leukemia as a Result of Pax5-Inherited Susceptibility. Cancer discovery 5, 1328-1343 (2015).

63. Nakayama, J. et al. BLNK suppresses pre-B-cell leukemogenesis through inhibition of JAK3. Blood 113, 1483-1492 (2009).

64. Heizmann, B., Kastner, P. \& Chan, S. Ikaros is absolutely required for pre-B cell differentiation by attenuating IL-7 signals. The Journal of experimental medicine 210, 2823-2832 (2013).

65. Katerndahl, C.D.S. et al. Antagonism of B cell enhancer networks by STAT5 drives leukemia and poor patient survival. Nature immunology 18, 694-704 (2017).

66. Cheng, Y. et al. LNK/SH2B3 regulates IL-7 receptor signaling in normal and malignant Bprogenitors. J Clin Invest 126, 1267-1281 (2016).

67. Dibirdik, I. et al. Engagement of interleukin-7 receptor stimulates tyrosine phosphorylation, phosphoinositide turnover, and clonal proliferation of human T-lineage acute lymphoblastic leukemia cells. Blood 78, 564-570. (1991).

68. Herranz, D. et al. A NOTCH1-driven MYC enhancer promotes T cell development, transformation and acute lymphoblastic leukemia. Nat Med 20, 1130-1137 (2014).

69. Loosveld, M. et al. MYC fails to efficiently shape malignant transformation in T-cell acute lymphoblastic leukemia. Genes, chromosomes \& cancer 53, 52-66 (2014).

70. Fistonich, C. et al. Cell circuits between B cell progenitors and IL-7(+) mesenchymal progenitor cells control B cell development. The Journal of experimental medicine 215, 2586-2599 (2018).

71. Swainson, L. et al. IL-7-induced proliferation of recent thymic emigrants requires activation of the PI3K pathway. Blood 109, 1034-1042 (2007).

72. Rathmell, J.C., Farkash, E.A., Gao, W. \& Thompson, C.B. IL-7 enhances the survival and maintains the size of naive T cells. $J$ Immunol 167, 6869-6876 (2001).

73. Barata, J.T., Cardoso, A.A., Nadler, L.M. \& Boussiotis, V.A. Interleukin-7 promotes survival and cell cycle progression of T-cell acute lymphoblastic leukemia cells by downregulating the cyclin-dependent kinase inhibitor p27(kip1). Blood 98, 1524-1531. (2001).

74. Barata, J.T. et al. Activation of PI3K Is Indispensable for Interleukin 7-mediated Viability, Proliferation, Glucose Use, and Growth of T Cell Acute Lymphoblastic Leukemia Cells. The Journal of experimental medicine 200, 659-669 (2004).

75. Ribeiro, D. et al. STAT5 is essential for IL-7-mediated viability, growth, and proliferation of T-cell acute lymphoblastic leukemia cells. Blood Adv 2, 2199-2213 (2018).

76. Li, Y. et al. IL-7 Receptor Mutations and Steroid Resistance in Pediatric T cell Acute Lymphoblastic Leukemia: A Genome Sequencing Study. PLoS medicine 13, e1002200 (2016).

77. Zenatti, P.P. et al. Oncogenic IL7R gain-of-function mutations in childhood T-cell acute lymphoblastic leukemia. Nat Genet 43, 932-939 (2011).

78. Shochat, C. et al. Gain-of-function mutations in interleukin-7 receptor-\{alpha\} (IL7R) in childhood acute lymphoblastic leukemias. The Journal of experimental medicine 208, 901908 (2011).

79. Zhang, J. et al. The genetic basis of early T-cell precursor acute lymphoblastic leukaemia. Nature 481, 157-163 (2012). 
80. Treanor, L.M. et al. Interleukin-7 receptor mutants initiate early T cell precursor leukemia in murine thymocyte progenitors with multipotent potential. The Journal of experimental medicine 211, 701-713 (2014).

81. Yokoyama, K. et al. In vivo leukemogenic potential of an interleukin 7 receptor alpha chain mutant in hematopoietic stem and progenitor cells. Blood 122, 4259-4263 (2013).

82. Liu, Y. et al. The genomic landscape of pediatric and young adult T-lineage acute lymphoblastic leukemia. Nat Genet 49, 1211-1218 (2017).

83. Gu, Z. et al. PAX5-driven subtypes of B-progenitor acute lymphoblastic leukemia. Nat Genet 51, 296-307 (2019).

84. Shochat, C. et al. Novel activating mutations lacking cysteine in type I cytokine receptors in acute lymphoblastic leukemia. Blood (2014).

85. Weijenborg Campos, L. et al. Oncogenic basic amino acid insertions at the extracellular juxtamembrane region of IL7RA cause receptor hypersensitivity. Blood 133, 1259-1263 (2019).

86. Cramer, S.D. et al. Mutant IL-7Ralpha and mutant NRas are sufficient to induce murine T cell acute lymphoblastic leukemia. Leukemia 32, 1795-1882 (2018).

87. Delgado-Martin, C. et al. JAK/STAT pathway inhibition overcomes IL7-induced glucocorticoid resistance in a subset of human T-cell acute lymphoblastic leukemias. Leukemia 31, 2568-2576 (2017).

88. Richter-Pechanska, P. et al. Identification of a genetically defined ultra-high-risk group in relapsed pediatric T-lymphoblastic leukemia. Blood cancer journal 7, e523 (2017).

89. Williams, R.T., Roussel, M.F. \& Sherr, C.J. Arf gene loss enhances oncogenicity and limits imatinib response in mouse models of Bcr-Abl-induced acute lymphoblastic leukemia. Proc Natl Acad Sci U S A 103, 6688-6693 (2006).

90. Williams, R.T., den Besten, W. \& Sherr, C.J. Cytokine-dependent imatinib resistance in mouse BCR-ABL+, Arf-null lymphoblastic leukemia. Genes \& development 21, 2283-2287 (2007).

91. Brown, V.I. et al. Rapamycin is active against B-precursor leukemia in vitro and in vivo, an effect that is modulated by IL-7-mediated signaling. Proc Natl Acad Sci U S A 100, 1511315118 (2003).

92. Cattaruzza, L. et al. Functional coexpression of Interleukin (IL)-7 and its receptor (IL-7R) on Hodgkin and Reed-Sternberg cells: Involvement of IL-7 in tumor cell growth and microenvironmental interactions of Hodgkin's lymphoma. Int J Cancer 125, 1092-1101 (2009).

93. Kibe, R. et al. IL-7Ralpha deficiency in p53null mice exacerbates thymocyte telomere erosion and lymphomagenesis. Cell death and differentiation 19, 1139-1151 (2012).

94. Swaminathan, S. et al. Mechanisms of clonal evolution in childhood acute lymphoblastic leukemia. Nature immunology 16, 766-774 (2015).

95. Al-Rawi, M.A., Rmali, K., Watkins, G., Mansel, R.E. \& Jiang, W.G. Aberrant expression of interleukin-7 (IL-7) and its signalling complex in human breast cancer. Eur J Cancer 40, 494-502 (2004).

96. Suzuki, K. et al. Clinical impact of immune microenvironment in stage I lung adenocarcinoma: tumor interleukin-12 receptor beta2 (IL-12Rbeta2), IL-7R, and stromal FoxP3/CD3 ratio are independent predictors of recurrence. J Clin Oncol 31, 490-498 (2013).

97. Yang, J. et al. IL-7 splicing variant IL-7delta5 induces EMT and metastasis of human breast cancer cell lines MCF-7 and BT-20 through activation of PI3K/Akt pathway. Histochem Cell Biol 142, 401-410 (2014).

98. Boesch, M. et al. Interleukin 7-expressing fibroblasts promote breast cancer growth through sustenance of tumor cell stemness. Oncoimmunology 7, e1414129 (2018). 
99. Li, J., Liu, J., Mao, X., Tang, Q. \& Lu, H. IL-7 receptor blockade inhibits IL-17-producing gammadelta cells and suppresses melanoma development. Inflammation 37, 1444-1452 (2014).

100. Maeurer, M.J. et al. Interleukin-7 (IL-7) in colorectal cancer: IL-7 is produced by tissues from colorectal cancer and promotes preferential expansion of tumour infiltrating lymphocytes. Scand J Immunol 45, 182-192 (1997).

101. Al-Rawi, M.A., Rmali, K., Mansel, R.E. \& Jiang, W.G. Interleukin 7 induces the growth of breast cancer cells through a wortmannin-sensitive pathway. Br J Surg 91, 61-68 (2004).

102. Ming, J., Zhang, Q., Qiu, X. \& Wang, E. Interleukin 7/interleukin 7 receptor induce cFos/c-Jun-dependent vascular endothelial growth factor-D up-regulation: a mechanism of lymphangiogenesis in lung cancer. Eur J Cancer 45, 866-873 (2009).

103. Mengus, C. et al. Elevated levels of circulating IL-7 and IL-15 in patients with early stage prostate cancer. J Transl Med 9, 162 (2011).

104. Qu, H. et al. IL-7/IL-7 receptor axis stimulates prostate cancer cell invasion and migration via AKT/NF-kappaB pathway. Int Immunopharmacol 40, 203-210 (2016).

105. Sakre, N. et al. RICTOR amplification identifies a subgroup in small cell lung cancer and predicts response to drugs targeting mTOR. Oncotarget 8, 5992-6002 (2017).

106. Liang, W.S. et al. Genome-wide characterization of pancreatic adenocarcinoma patients using next generation sequencing. PLoS One 7, e43192 (2012).

107. Lin, D.C. et al. Genomic and molecular characterization of esophageal squamous cell carcinoma. Nat Genet 46, 467-473 (2014).

108. Shah, S.P. et al. The clonal and mutational evolution spectrum of primary triple-negative breast cancers. Nature 486, 395-399 (2012).

109. Seol, M.A. et al. Interleukin-7 Contributes to the Invasiveness of Prostate Cancer Cells by Promoting Epithelial-Mesenchymal Transition. Sci Rep 9, 6917 (2019).

110. Ming, J., Jiang, G., Zhang, Q., Qiu, X. \& Wang, E. Interleukin-7 up-regulates cyclin D1 via activator protein-1 to promote proliferation of cell in lung cancer. Cancer Immunol Immunother 61, 79-88 (2012).

111. Cui, L. et al. Overexpression of IL-7 enhances cisplatin resistance in glioma. Cancer Biol Ther 13, 496-503 (2012).

112. Roato, I. et al. Bone invading NSCLC cells produce IL-7: mice model and human histologic data. BMC Cancer 10, 12 (2010).

113. Rosenberg, S.A. et al. IL-7 administration to humans leads to expansion of CD8+ and CD4+ cells but a relative decrease of CD4+ T-regulatory cells. J Immunother 29, 313-319 (2006).

114. Sportes, C. et al. Administration of rhIL-7 in humans increases in vivo TCR repertoire diversity by preferential expansion of naive T cell subsets. The Journal of experimental medicine 205, 1701-1714 (2008).

115. Tredan, O. et al. ELYPSE-7: a randomized placebo-controlled phase IIa trial with CYT107 exploring the restoration of CD4+ lymphocyte count in lymphopenic metastatic breast cancer patients. Annals of oncology: official journal of the European Society for Medical Oncology 26, 1353-1362 (2015).

116. Merchant, M.S. et al. Adjuvant Immunotherapy to Improve Outcome in High-Risk Pediatric Sarcomas. Clin Cancer Res 22, 3182-3191 (2016).

117. Levy, D.S., Kahana, J.A. \& Kumar, R. AKT inhibitor, GSK690693, induces growth inhibition and apoptosis in acute lymphoblastic leukemia cell lines. Blood 113, 1723-1729 (2009).

118. Sereti, I. et al. IL-7 administration drives T cell-cycle entry and expansion in HIV-1 infection. Blood 113, 6304-6314 (2009). 
119. Sortino, O. et al. IL-7 treatment supports CD8+ mucosa-associated invariant T-cell restoration in HIV-1-infected patients on antiretroviral therapy. AIDS 32, 825-828 (2018).

120. Sheikh, V. et al. Administration of interleukin-7 increases CD4 T cells in idiopathic CD4 lymphocytopenia. Blood 127, 977-988 (2016).

121. Patel, A., Patel, J. \& Ikwuagwu, J. A case of progressive multifocal leukoencephalopathy and idiopathic CD4+ lymphocytopenia. The Journal of antimicrobial chemotherapy 65, 2697-2698 (2010).

122. Alstadhaug, K.B. et al. Treatment of progressive multifocal leukoencephalopathy with interleukin 7. JAMA Neurol 71, 1030-1035 (2014).

123. Hotchkiss, R.S. et al. Sepsis and septic shock. Nature reviews. Disease primers 2, 16045 (2016).

124. Thampy, L.K. et al. Restoration of T Cell function in multi-drug resistant bacterial sepsis after interleukin-7, anti-PD-L1, and OX-40 administration. Plos One 13, e0199497 (2018).

125. Francois, B. et al. Interleukin-7 restores lymphocytes in septic shock: the IRIS-7 randomized clinical trial. JCI Insight 3 (2018).

126. Cramer, S.D., Aplan, P.D. \& Durum, S.K. Therapeutic targeting of IL-7Ralpha signaling pathways in ALL treatment. Blood 128, 473-478 (2016).

127. Kern, B., Li, W., Bono, C., Lee, L.F. \& Kraynov, E. Receptor occupancy and blocking of STAT5 signaling by an anti-IL-7 receptor alpha antibody in cynomolgus monkeys. Cytometry. Part B, Clinical cytometry 90, 191-198 (2016).

128. Belarif, L. et al. IL-7 receptor blockade blunts antigen-specific memory T cell responses and chronic inflammation in primates. Nat Commun 9, 4483 (2018).

129. Akkapeddi, P. et al. A fully human anti-IL-7Ralpha antibody promotes antitumor activity against T-cell acute lymphoblastic leukemia. Leukemia (2019).

130. Senkevitch, E. et al. Inhibiting Janus Kinase 1 and BCL-2 to treat T cell acute lymphoblastic leukemia with IL7-Ralpha mutations. Oncotarget 9, 22605-22617 (2018). 


\section{Figure Legends}

Figure 1. Atlas of IL-7 production. IL-7 is produced in both primary (bone marrow and thymus) and secondary (lymph nodes and spleen) lymphoid organs where it supports development and maintenance of lymphocyte populations. However, production is also detected in non-lymphoid sites such as mucosal and epithelial layers, where it is important for maintaining intact barrier, as well as other sites such as the brain, where function is less clear.

Figure 2. Developmental functions of IL-7. During hematopoiesis, IL-7 signaling is important in supporting development of multiple lymphoid lineages. IL-7 signaling promotes commitment of progenitors to B-cell fate, but is also essential for development of group 2 and group 3 ILCs in the bone marrow. In the thymus, IL-7 signaling promotes $\gamma \delta \mathrm{T}$ cell lineage development but is also essential to support expansion of DN4 $\alpha \beta$ thymocytes as they proliferate and fill the thymus with DP thymocytes ready for selection.

Figure 3. Potential mechanisms promoting the outgrowth of leukemia cells in the context of IL-7 stimulation. Several scenarios may explain how a limited resource such as IL-7 may benefit more (pre)leukemic cells than normal lymphoid precursors. (a) Maturation arrest is a characteristic of both B- and T-ALL cells that protects them from stringent selection steps that necessarily occur as a lymphoid precursor differentiates in the bone marrow or thymus, while fully benefiting from the survival and proliferative signals arising from IL-7 signaling. (b) Oncogenic hits such as MYC activation often lead to cancer development only if complemented by secondary hits that counterbalance oncogene-induced apoptosis. IL-7 provides non-cell-autonomous pro-survival cues that should allow (pre)leukemic cells to thrive, buying time for subsequent fully-transforming lesions to occur and subsequent (more aggressive) leukemia development. (c) Different genetic 
lesions such as intracellular Notch (ICN1), ZEB2 translocation (tZEB2) or mutations in ribosomal genes (RPLmut) in T-ALL, or SH2B3 or PAX5 deficiency in B-ALL, lead to IL-7R $\alpha$ upregulation and consequent IL-7 hypersensitivity. (d) On the other hand, (pre)leukemic cells can downregulate the production of IL-7 by stromal cells in such a way that normal precursors fail to fully access an essential factor for their development, while full transformation or hyperresponsiveness to IL-7 may allow for expansion of the malignant cells in low IL-7-containing niches. (e) IL-7 signaling differs subtly between normal T-cells and T-ALL cells. STAT5 and PI3K/Akt/mTOR signaling are both absolutely required for T-ALL cell survival and cell cycle progression - although, in contrast to normal T cells, STAT5 does not appear to upregulate Bcl-2 downstream from IL-7 stimulation. IL-7 promotes cell cycle progression in normal T-cells via PI3K/Akt/mTOR signaling and viability via STAT5.

Figure 4. Classes of $I L 7 R$ gain-of-function mutants in leukemia. (a) IL-7R $\alpha$ mutants found in leukemia patients fall into 4 classes, affecting either exon 6 (Type 1) or, less frequently, exon 5 (Type 2). The latter are all S185C variants affecting the trasmembrane domain and occur exclusively in B-ALL patients. Mutations affecting exon 6 are: type 1a) insertions or insertionsdeletions introducing an unpaired cysteine in the extracellular juxtamembrane-transmembrane (EJMT) region; type 1b) lie deeper in the transmembrane domain region and include tryptophans, SxxxG-related motifs, or other residues; type 1c) occur in the EJMT region but lead to the introduction of negatively-charged amino acids such as arginine (R) or histidine (H). (b) Specificities on receptor dimerization and signaling resulting from each class of mutations.

Figure 5. Potential pro-tumoral functions of IL-7R-mediated signaling in solid cancers. In vitro and in vivo studies suggest that the IL-7/IL-7R axis is implicated in promoting proliferation, epithelial-mesenchymal transition (EMT), migration, invasion and metastasis, as well as resistance 
to chemotherapy of cancer cells, while also stimulating osteoclastogenesis in bone metastasis and lymphangiogenesis. In agreement, $I L 7 R$ is amplified in different cancers and high levels of IL-7 and/or IL-7R can be associated with poor prognosis. 
Figure 1

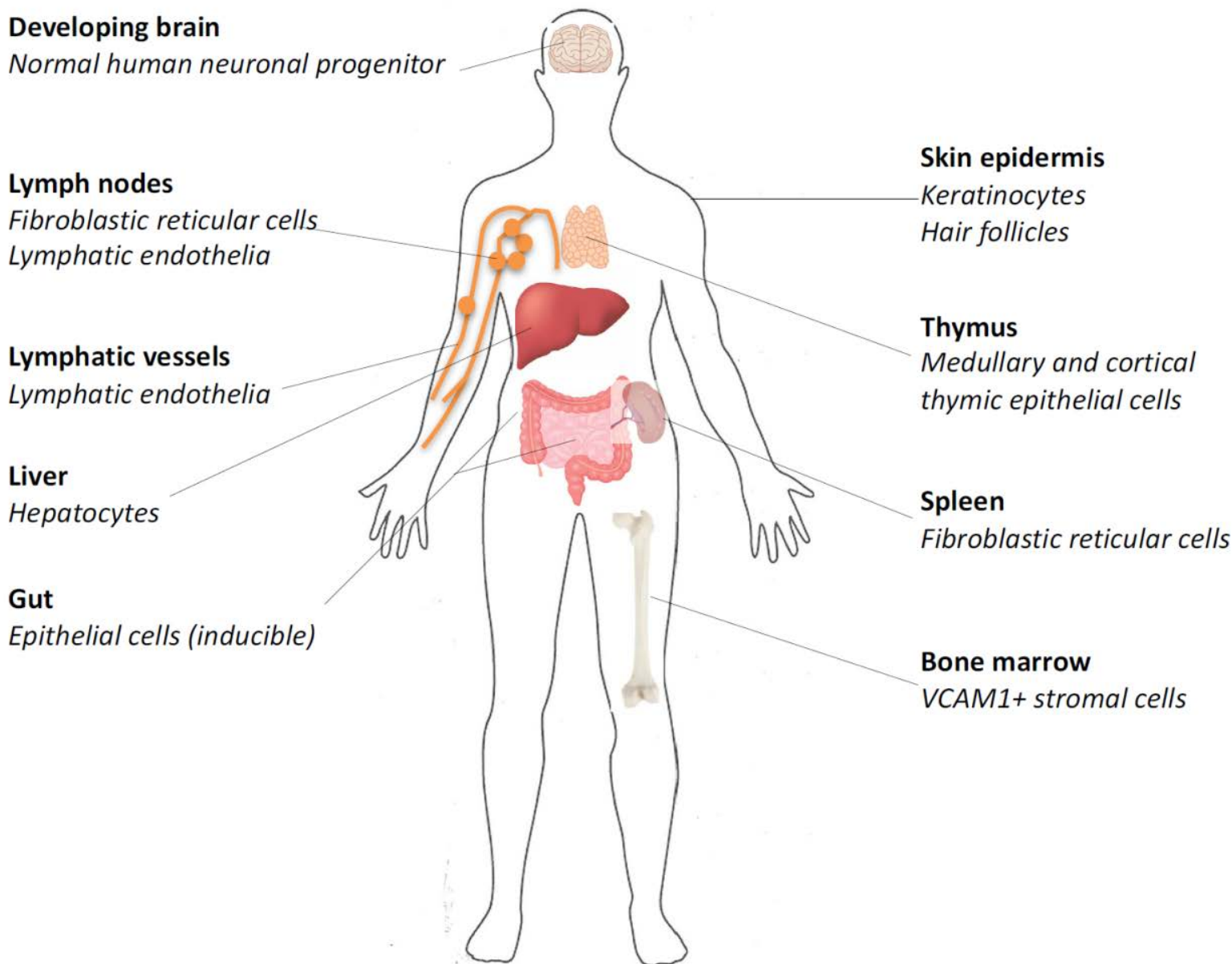


Figure 2

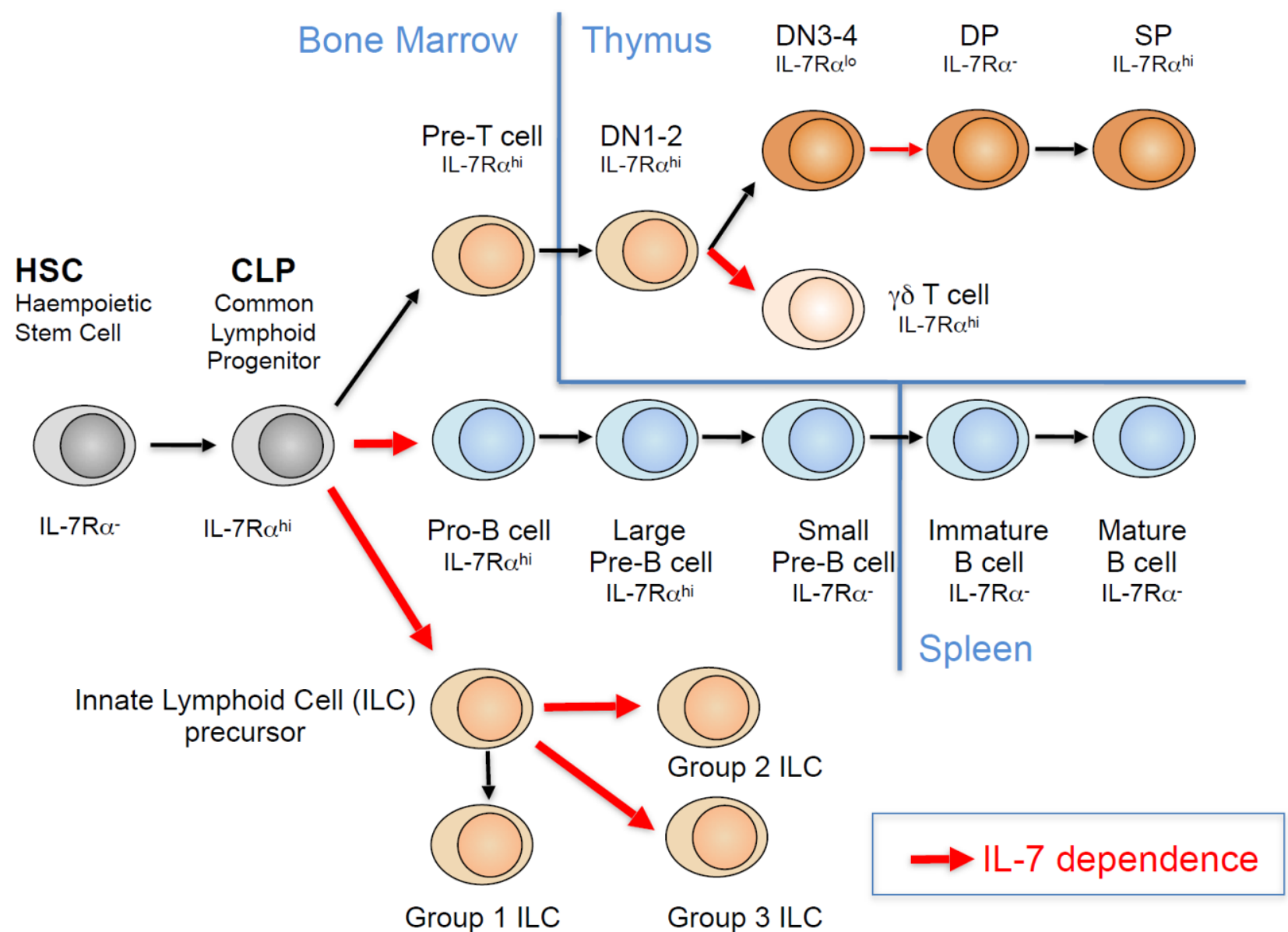




\section{Figure 3}

a

Normal lymphoid cell (prone to differentiate)

IL-7
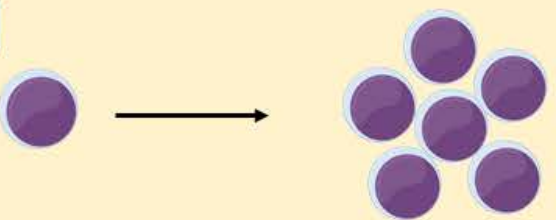

Differentiation
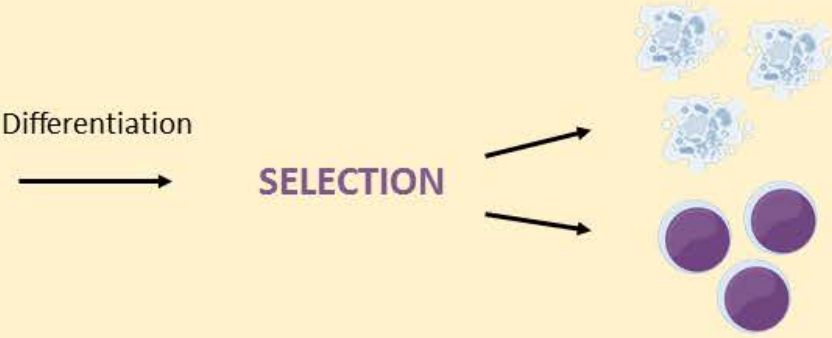

Leukemic cell (developmentally blocked)

IL-7

Oncogenic hit1 (e.g. MYC)

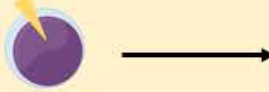

Oncogenic hit1 (e.g. MYC)

IL-7
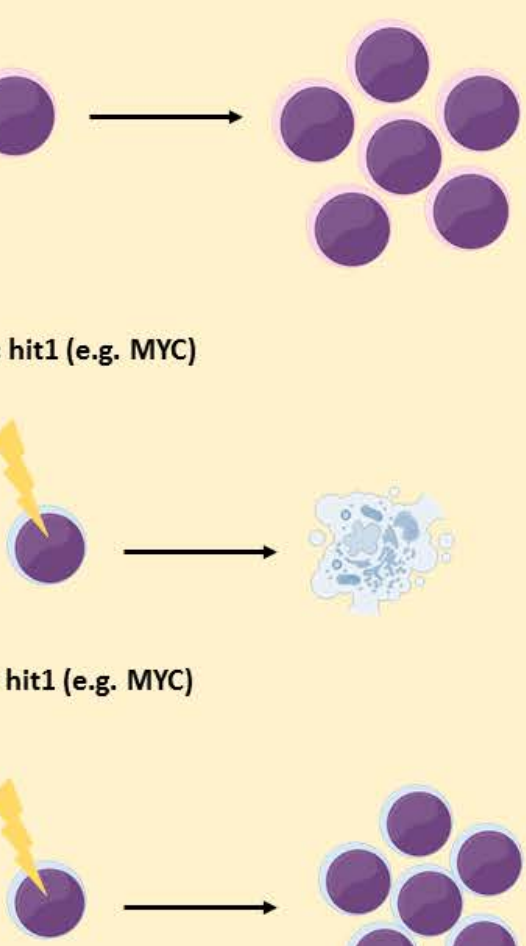

Differentiation
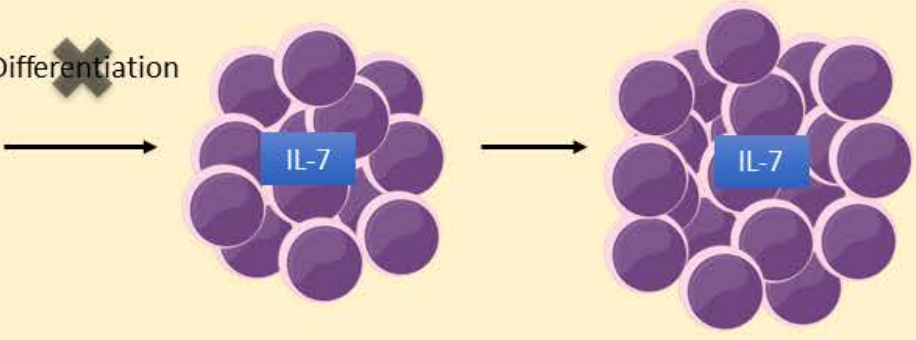

Oncogenic hit2

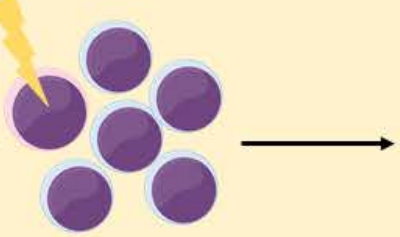

Leukemia development

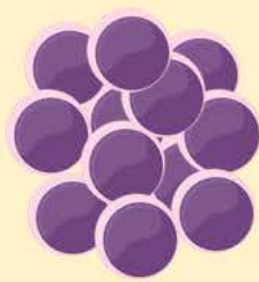

T-ALL

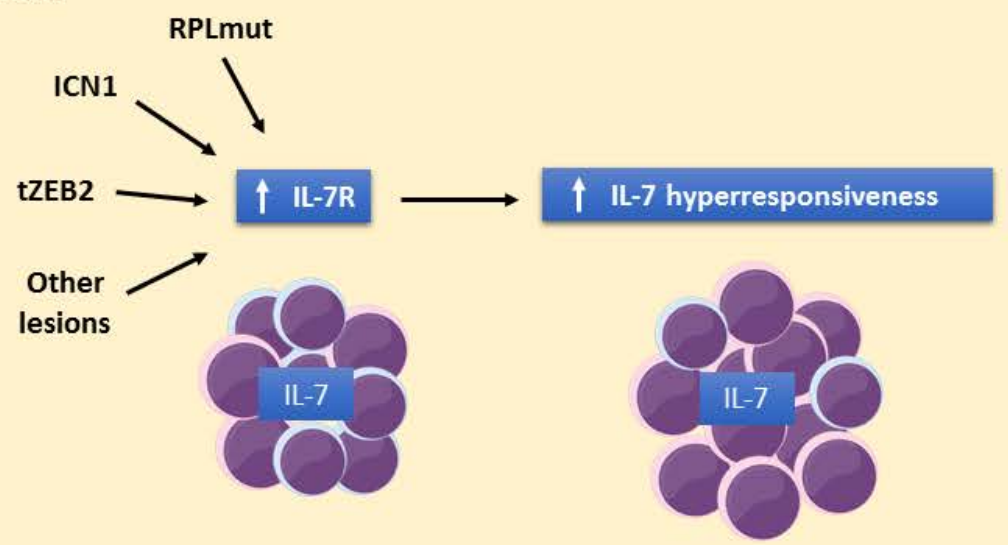


d

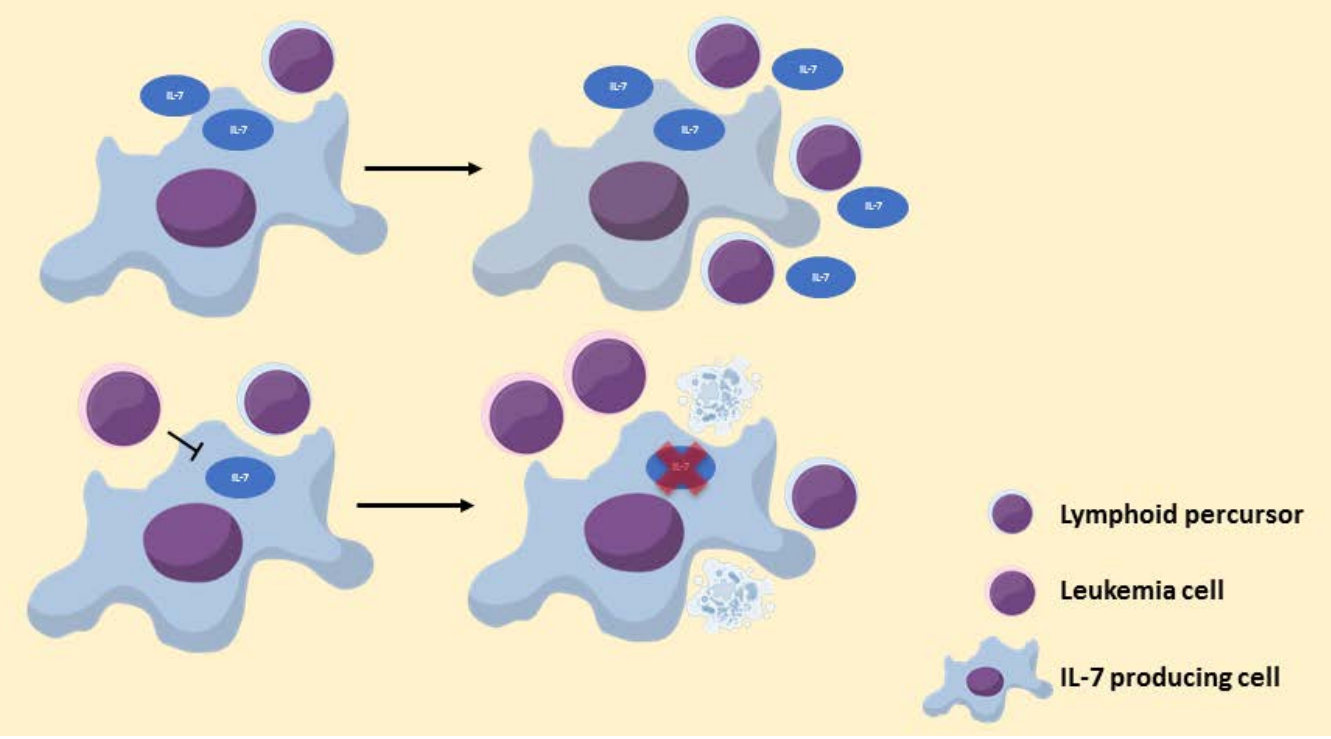

e

Normal T-cell

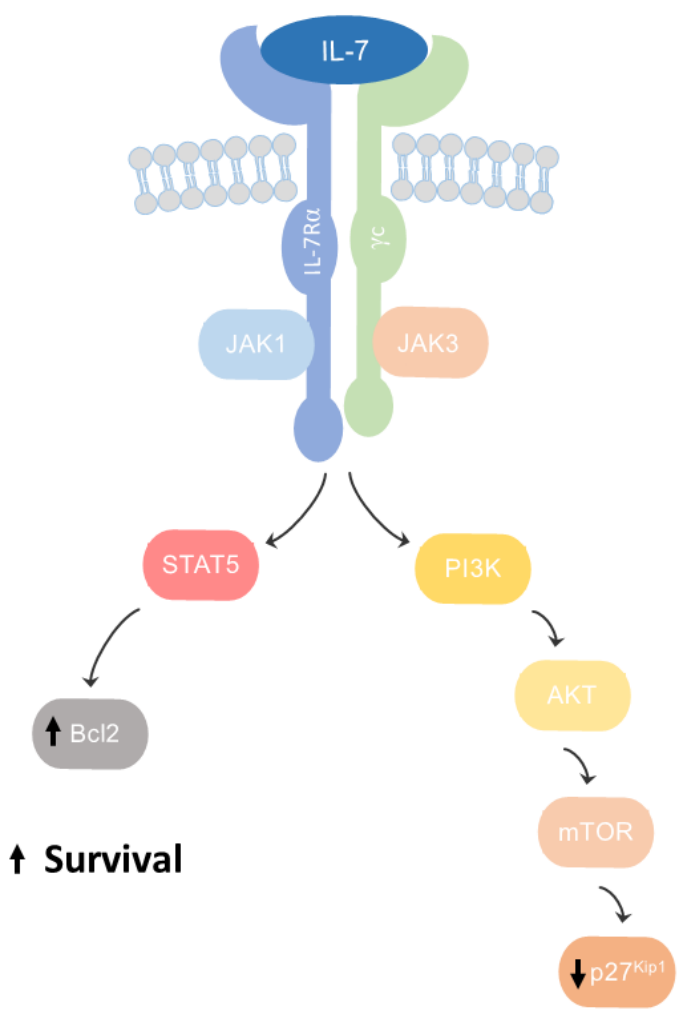

$\uparrow$ Cell cycle
T-ALL cell

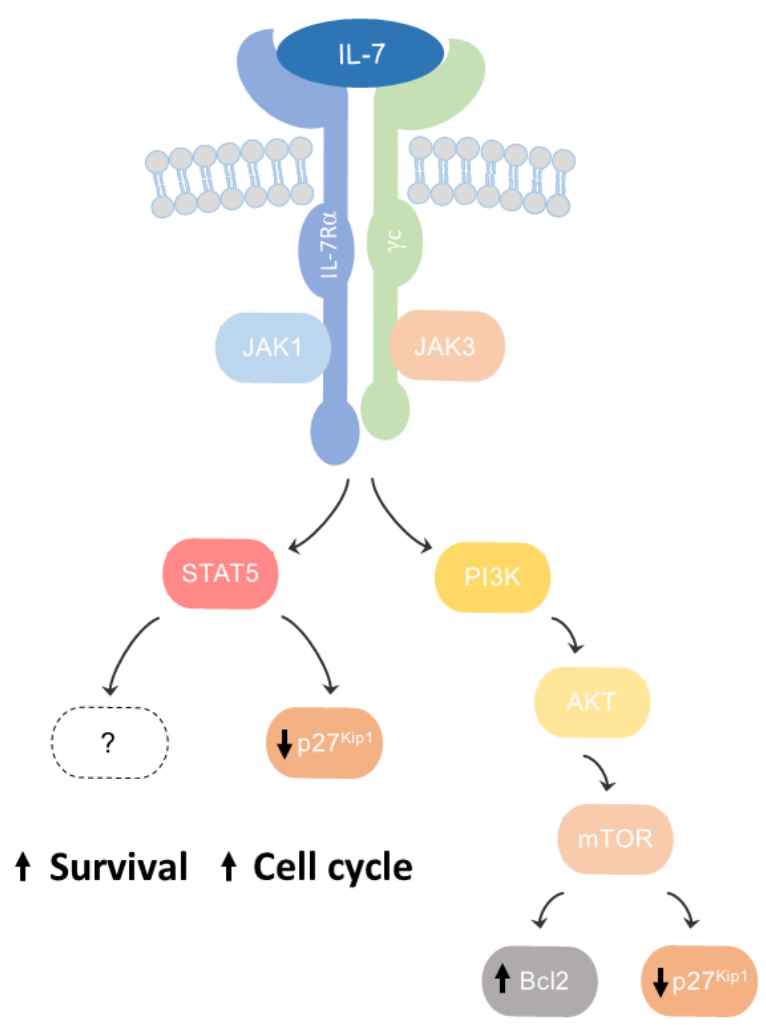

$\uparrow$ Survival $\uparrow$ Cell cycle 
Figure 4

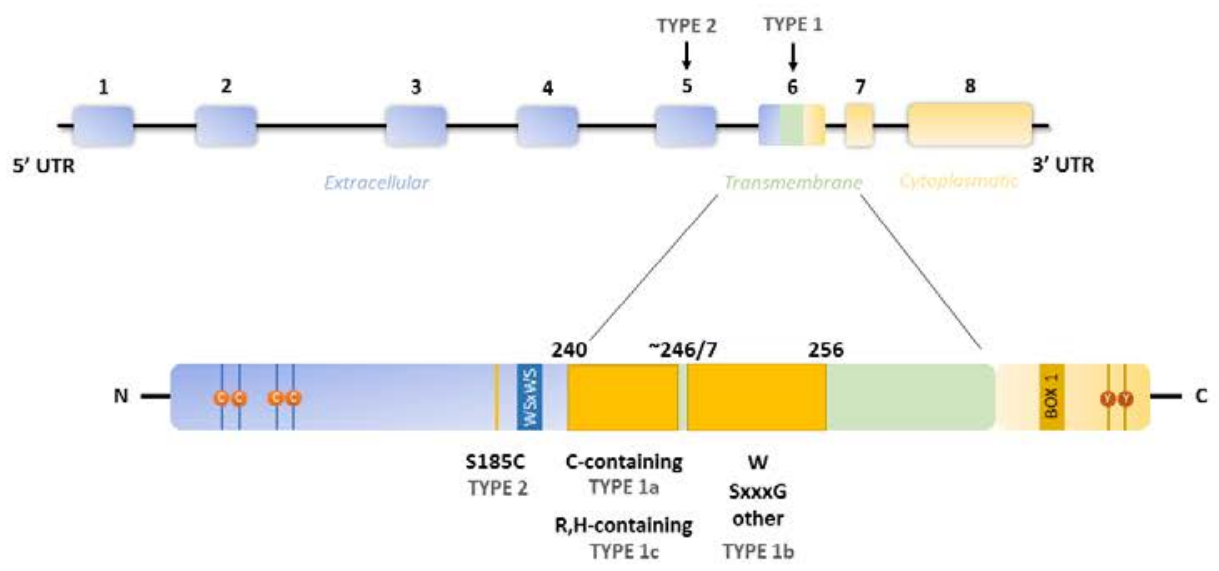

b

TYPE 1a

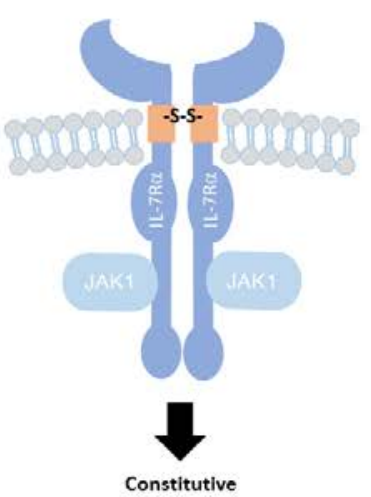

TYPE 1c

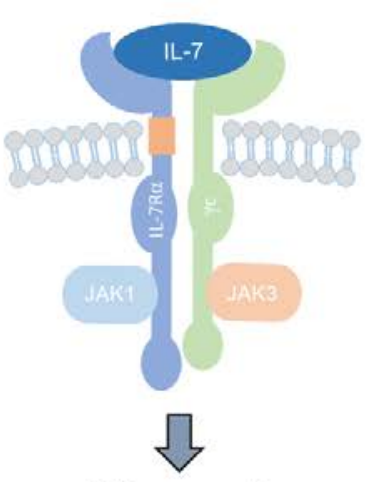

IL-7 hyper-responsive
TYPE 1b

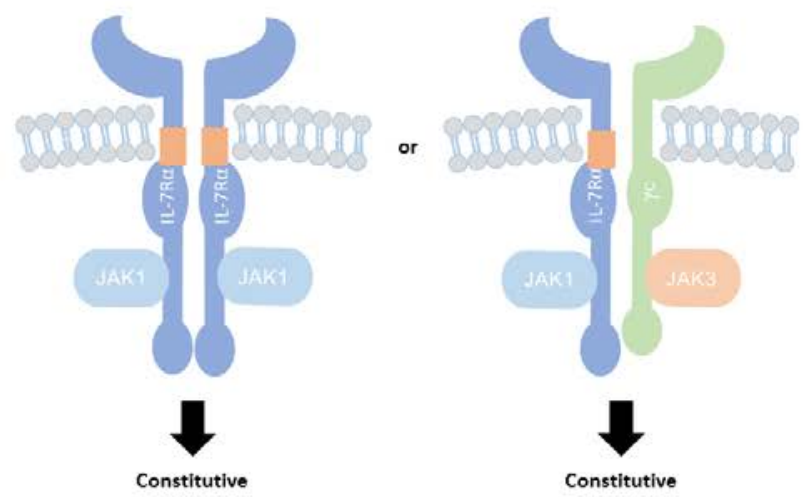

TYPE 2

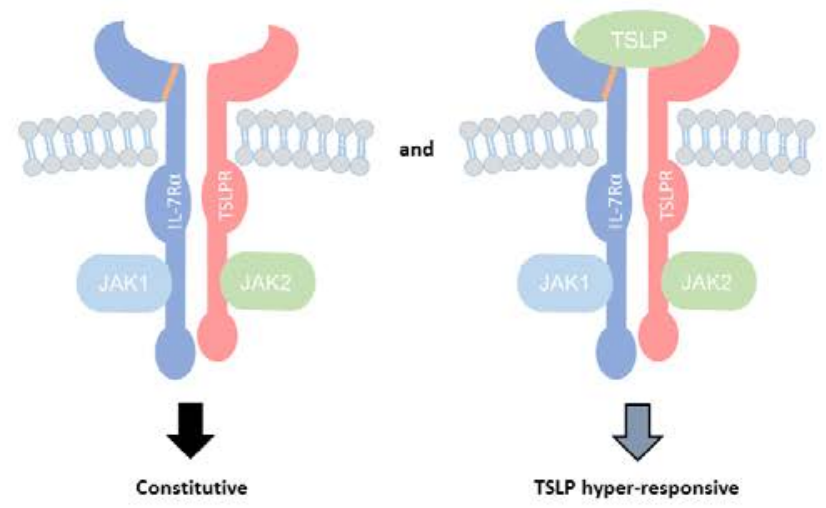




\section{Figure 5}

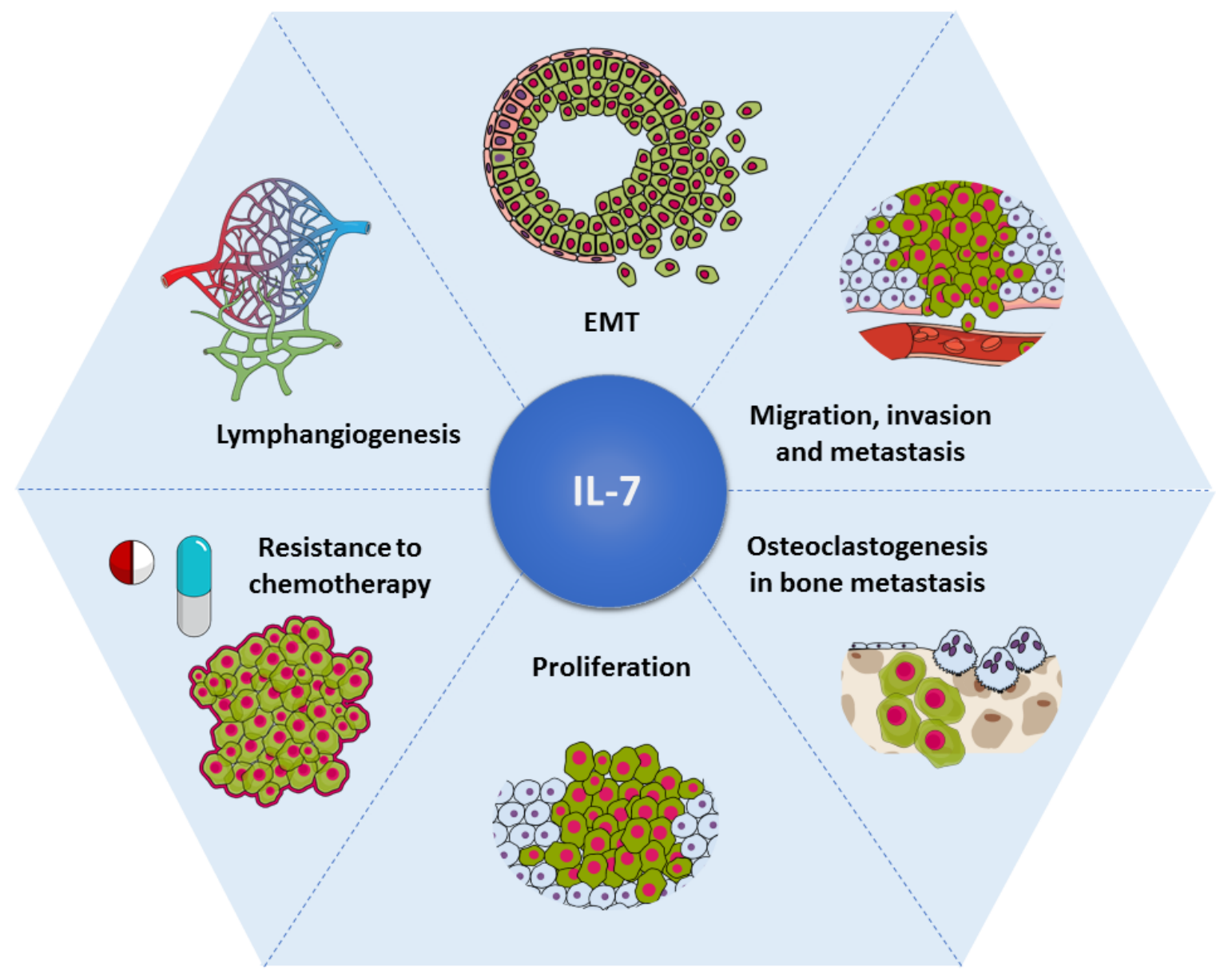


Table 1. IL-7/IL-7R agonists and antagonists in human subjects

\begin{tabular}{llll} 
Product & Source & Disease & Current/future trials \\
\hline IL-7 & Revimmune (Cytheris) & Cancers & CITN-03, NCT03513952 \\
& & HSCT & NCT03600896 \\
& HIV & \\
& & CD4 deficiency & \\
& & Myco, MDR & future \\
& & PML & \\
IL-7-FC & Neolmmune Tech & Sepsis & NCT03821038 \\
& & Cancers & current and future \\
& & Elderly & plus vaccine - future \\
alL-7R & Pfizer & T1D, MS & current \\
& GSK & Sjogren's & current \\
& OSE & IBD, Sjogren's & future \\
& NCl/Fannin & ALL & future \\
\hline
\end{tabular}

Abbreviations: Hematopoietic stem cell transplantation (HSCT), human immunodeficiency virus (HIV), mycobacterial infection in AIDS patients (Myco), multi-drug resistant bacterial infections (MDR), progressive multifocal leukoencephalopathy (PML), type 1 diabetes (T1D), multiple sclerosis (MS), inflammatory bowel disease (IBD), acute lymphoblastic leukemia (ALL). 